\title{
Analysis of differentially expressed genes between rheumatoid arthritis and osteoarthritis based on the gene co-expression network
}

\author{
QING-YOU LU*, QING-HUI HAN*, XIA LI, ZENG-CHUN LI, YU-TAO PAN, \\ LIN LIU and QING-GE FU \\ Department of Trauma Surgery, East Hospital Affiliated to Tongji University, Shanghai 200120, P.R. China
}

Received August 11,2013; Accepted March 5, 2014

DOI: $10.3892 / \mathrm{mmr} .2014 .2166$

\begin{abstract}
The aim of the current study was to investigate disease-associated genes and related molecular mechanisms of osteoarthritis (OA) and rheumatoid arthritis (RA). Using GSE7669 datasets downloaded from Gene Expression Omnibus databases, the differentially expressed genes (DEGs) between RA and OA synovial fibroblasts (SFBs) $(\mathrm{n}=6$ each) were screened. DEG-associated co-expression and topological properties were analyzed to determine the rank of disease-associated genes. Specifically, the fold change of differentially expressed genes, the clustering coefficient and the degree of differential gene co-expression were integrated to determine the disease-associated gene ranking. The underlying molecular mechanisms of these crucial disease-associated genes were investigated by gene ontology (GO) enrichment analysis. A total of 1313 DEGs, including 1068 upregulated genes and 245 downregulated genes were observed. The top 20 disease-associated genes were identified, including proteoglycan 4 , inhibin $\beta$ B, carboxypeptidase $\mathrm{M}$, alcohol dehydrogenase $1 \mathrm{C}$ and integrin $\beta 2$. The major GO biological processes of these top 20 disease-associated genes were highly involved in the immune system, such as responses to stimuli, immune responses and inflammatory responses. This large-scale gene expression study observed disease-associated genes and their associated GO function in RA and OA, which may provide opportunities for biomarker development and novel insights into the molecular mechanisms of these two diseases.
\end{abstract}

Correspondence to: Dr Xia Li or Dr Zeng-Chun Li, Department of Trauma Surgery, East Hospital Affiliated to Tongji University, 150 Jimo Road, Shanghai 200120, P.R. China

E-mail: lixiallxx@hotmail.com

E-mail: jlijianli@126.com

${ }^{*}$ Contributed equally

Key words: differentially expressed genes, molecular mechanisms, gene ontology

\section{Introduction}

Human rheumatoid arthritis (RA), a polyarticular disease of autoimmune nature (1), is perpetuated by an invasive pannus tissue, whereas osteoarthritis (OA), as a non-inflammatory degenerative disease of the articular cartilage (2), is characterized by an increased tendency for novel blood vessel formation $(3,4)$. Furthermore, recent studies have reported information regarding the differences in pathogenesis between RA and OA. Patients with RA present with joint destruction caused by hyperplasia of the synovial lining, infiltration of mononuclear cells into the sublining layer, stimulation of fibroblast-like synoviocytes and the increase of catabolic mediators, including interleukin (IL)- $1 \beta$ tumor necrosis factor (TNF)- $\alpha$ and matrix metalloproteinases (MMP) (5). By contrast, in patients with OA, joint destruction is due to cartilage degradation and elevated concentrations of cartilage matrix components, which elicit the presence of synovitis. In addition, synovitis aggravates the damage of articular cartilage by releasing inflammatory cytokines and destructive proteases (6).

Although OA and RA have different modes of pathogenesis, the current treatment of these two disease is similar, including nonsteroidal anti-inflammatory drugs, applied for pain and inflammation management $(7,8)$; disease-modifying antirheumatic drugs, which function as a classical first-line therapy to minimize or prevent joint damage $(7,9)$; and surgical treatment performed to replace the joints $(10,11)$. However, these approaches induce a number of adverse events and less than satisfactory clinical outcomes $(12,13)$. Thus, disease-specific therapy requires further investigation.

Genetic factors are also critical in the pathogenesis of RA and OA. Bramlage et al (14) identified that bone morphogenetic protein (BMP)-4 and BMP-5 were downregulated in $\mathrm{OA}$ and RA compared with that expressed in normal synovial tissue, suggesting a role of distinct BMPs in joint homeostasis, which may be altered in inflammatory and degenerative joint diseases. In addition, Pohlers et al (15) demonstrated that upregulation of the tumor growth factor (TGF)- $\beta$ pathway was observed in RA synovial fibroblasts (SFBs), resulting in significant overexpression of MMP-11 mRNA and protein in RA SFBs, but not in OA SFBs. However, differentially expressed 
genes (DEGs) and molecular mechanisms underlying RA and $\mathrm{OA}$ are not yet fully understood.

In the current study, comparative analysis of DEG characteristics between RA and OA profiles was performed to identify DEGs with potential pathophysiological relevance. Differential gene co-expression networks were constructed and analyzed to identify disease candidate genes.

\section{Materials and methods}

Microarray data analysis. The gene expression data was downloaded from the Gene Expression Omnibus (GEO) database (http://www.ncbi.nlm.nih.gov/geo/) using GEO accession no. GSE7669 (15). The database contains six RA and six OA SFBs derived from a human study using the Affymetrix Human Genome U95 version 2 (Affymetrix Inc., Santa Clara, CA, USA) platform. Background-corrected signal intensities were determined using the MAS 5.0 software (Affymetrix ${ }^{\circledR}$ ). The normalization of datasets obtained on Affymetrix arrays was performed using the preprocessCore package in $\mathrm{R}(16)$.

Screening DEGs. Significance analysis of microarray (SAM) is widely used to detect genes on a microarray with statistically significant changes in expression (17). As an alternative to a t-test, significance SAM4.0 was employed for determination of differentially expressed genes between RA and OA SFBs and the threshold was set as log Ifold changel $\geq 2$.

Constructing differential gene co-expression networks. Prior to the construction of co-expression networks, the expression value was determined from differentially expressed gene samples between RA and OA. Next, Pearson's coefficient was applied to calculate correlations between DEGs. The differential co-expressiond network was then constructed as the threshold was set as $r \geq 0.8$.

Network topological analysis. The Cytoscape plug-in Network Analyzer (18) was used for network visualization and gene co-expression analysis. For each network the number of nodes and edges was simply calculated. The clustering coefficient of a network $C_{n}$ was counted as the average clustering coefficient of all of its non-singleton nodes via the formula: $\mathrm{C}_{\mathrm{n}}=2 \mathrm{e}_{\mathrm{n}} / \mathrm{k}_{\mathrm{n}}(\mathrm{kn}-1)(1)$; where $\mathrm{e}_{\mathrm{n}}$ denotes the number of edges between the $k_{n}$ neighbors of $n$ and $k_{n}$ is the degree of $n(19)$.

Gene ranking. Conventionally, disease-associated gene ranking is determined by linkage analysis and gene expression profile analysis (20-22). However, these methods are mostly limited to a single statistic indicator. In the current study, the fold change of differentially expressed genes, the clustering coefficient and the degree of differential gene co-expression were integrated to determine the disease-associated gene ranking using the formula (2): $\mathrm{Cri}_{\mathrm{n}}=\mathrm{FC}_{\mathrm{n}}+$ degree $_{\mathrm{n}}+\mathrm{C}_{\mathrm{n}}$ (2); where $\mathrm{Cri}_{\mathrm{n}}$ is an indicator of the gene ranking (the larger the value is, the higher the gene ranks), $\mathrm{FC}_{\mathrm{n}}$ stands for the value of gene fold change, degree $_{\mathrm{n}}$ and $\mathrm{C}_{\mathrm{n}}$ denotes the average degree and the clustering coefficient of a co-expression network respectively.

Calculating GO enrichment. Biological function and candidate gene-associated biological pathways can be determined

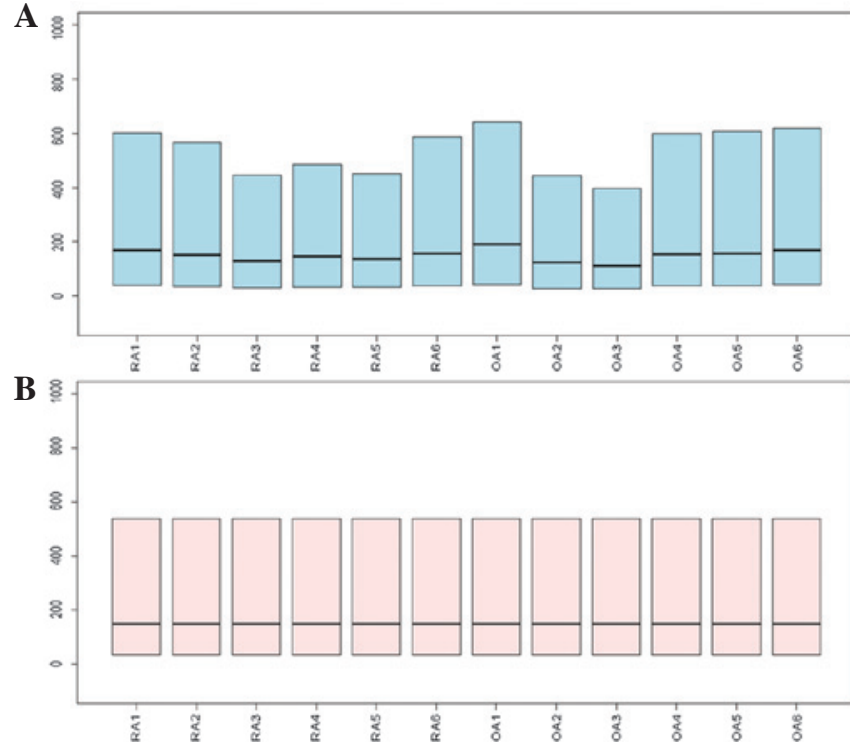

Figure 1. Normalization of samples. (A) Prior to normalization and (B) following normalization, RA1-6 $=6$ RA samples, OA1- $6=6$ OA samples. RA, rheumatoid arthritis; OA, osteoarthritis.

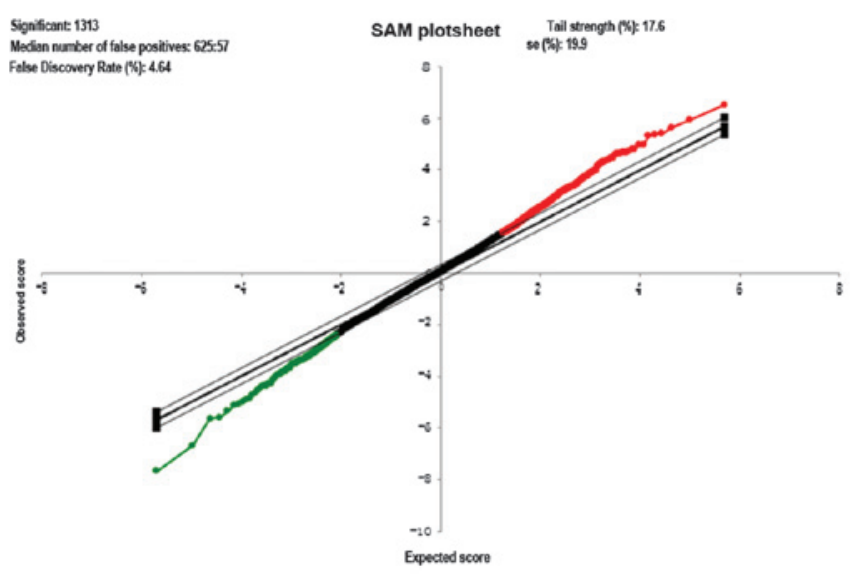

Figure 2. SAM plotsheet outputs. The red, green and black dots indicate upregulated, downregulated and insignificant genes, respectively. The upper and lower $45^{\circ}$ lines indicate the $\Delta$ threshold boundaries. Tail length (17.6\%) and standard error (se) rate $(19.9 \%)$ is shown at the upper right corner of the plotsheet. The number of significant genes (1313), median number of false positives (625.57) and false discovery rate (4.64\%) are shown at the upper left corner of the plotsheet. SAM, significance analysis of microarray.

by GO (http://www.geneontology.org). The open access software DAVID (23) was used to access the GO enrichment of candidate genes, which is a slightly modified Fisher's exact test, identical to the EASE score (24).

\section{Results}

Differentially gene expression. Gene expression in six RA SFBs was compared with that in six OA SFBs. The R preprocessCore was used to normalize and preprocess the presented data ( $\log$ Ifold changel $\geq 2$ ) (Fig. 1). A total of 1313 differentially expressed genes were observed, in which 1068 genes were upregulated and 245 genes were downregulated in OA SFBs compared with RA SFBs (Fig. 2). 
Table I. genes with the highest node degrees in the co-expression network.

\begin{tabular}{|c|c|c|c|}
\hline No. & Gene ID & Degree & $\begin{array}{l}\text { Clustering } \\
\text { coefficient }\end{array}$ \\
\hline 1 & TBCD & 190 & 0.3267613 \\
\hline 2 & ABCA3 & 169 & 0.3709495 \\
\hline 3 & DDAH2 & 164 & 0.3754302 \\
\hline 4 & SLC29A1 & 164 & 0.3787221 \\
\hline 5 & ARL2 & 162 & 0.3647726 \\
\hline 6 & MAPKAPK3 & 161 & 0.3473602 \\
\hline 7 & GUK1 & 160 & 0.3463050 \\
\hline 8 & GNB5 & 159 & 0.3894594 \\
\hline 9 & C16orf45 & 154 & 0.3922417 \\
\hline 10 & PPP1R7 & 152 & 0.3580515 \\
\hline \multicolumn{4}{|c|}{$\begin{array}{l}\text { TBCD, tubulin folding cofactor D; ABCA3, ATP-binding } \\
\text { cassette sub-family A member 3; DDAH2, dimethylargining dimeth- } \\
\text { lyamnohydrolase 2; SLC29A1, solute carrier family 29; ARL2, } \\
\text { ADP-ribosylation factor-like 2; MAPKAPK3, mitogen-activated } \\
\text { protein kinase-activated protein kinase 3; GUK1, guanylate kinase 1; } \\
\text { GNB5, guanine nucleotide binding protein; C16orf45, chromosome } \\
16 \text { open reading frame } 45 \text { [Homo sapiens (human)]; PPP1R7, protein } \\
\text { phosphatase 1, regulatory subunit } 7 \text {. }\end{array}$} \\
\hline
\end{tabular}

Differential co-expression network. To construct a differential gene co-expression network, data from a total 1313 DEGs were extracted from the expression profile and Pearson's coefficient was applied to calculate correlations between these DEGs (the threshold as $r \geq 0.8$ ). A total 1302 nodes and 20372 edges were identified among the differential gene co-expression network, which is comprised of two closely connected sub-networks (Fig. 3)

Top 10 genes with the highest node degrees in the co-expression network. Clustering coefficient and degree were analyzed to detect the importance of disease-associated genes from differential gene co-expression networks. In the current study, the top 10 degree of corresponding genes are shown in Table I. Briefly, tubulin folding cofactor, ATP-binding cassette sub-family A member 3 and dimethylargining dimethlyamnohydrolase 2 were the top three genes of the list, with a considerably higher degree in the co-expression network.

Top 20 disease candidate genes. To determine the association between DEGs and the two diseases, OA and RA, disease candidate genes were ranked according to the $\mathrm{Cri}_{n}$ value using the formula $\mathrm{Cri}_{n}=\mathrm{FC}_{\mathrm{n}}+$ degree $_{\mathrm{n}}+\mathrm{C}_{\mathrm{n}}$. As presented in Table II, the top 5 of 20 disease candidate genes were inhibin $\beta$ B (INHBB), carboxypeptidase $\mathrm{M}$, alcohol dehydrogenase 1A, integrin $\beta 2$ (ITGB2) and collagen, type XI, $\alpha 1$ (COL11A1), respectively.

Functional annotation of candidate genes. The analysis of GO enrichment was used to detect the association between the top 20 disease-associated candidate genes and associated biological processes and pathways $(\mathrm{P}<0.05)$. Overall, the majority of the top 20 disease-associated candidate genes, including INHBB,
Table II. Top 20 disease candidate genes.

\begin{tabular}{|c|c|c|c|}
\hline Rank & Symbol & Regulation & $\mathrm{Cri}_{\mathrm{n}}$ \\
\hline 1 & INHBB & OA (down) & 10.10852052 \\
\hline 2 & CPM & OA (down) & 8.60116868 \\
\hline 3 & ADH1C & OA (up) & 6.83154569 \\
\hline 4 & ITGB2 & OA (down) & 6.62193398 \\
\hline 5 & COL11A1 & OA (down) & 6.28105739 \\
\hline 6 & ADH1A & OA (up) & 6.23549718 \\
\hline 7 & C10orf116 & OA (up) & 5.89913165 \\
\hline 8 & SLC29A1 & OA (up) & 5.84062491 \\
\hline 9 & PRG4 & OA (up) & 5.57878155 \\
\hline 10 & CHL1 & OA (up) & 5.54248666 \\
\hline 11 & RARRES2 & OA (down) & 5.40151339 \\
\hline 12 & MS4A2 & OA (down) & 5.36385476 \\
\hline 13 & CCR3 & OA (up) & 5.05797001 \\
\hline 14 & IFIT2 & OA (up) & 4.88706770 \\
\hline 15 & RSAD2 & OA (up) & 4.77719664 \\
\hline 16 & CLIC5 & OA (up) & 4.77298747 \\
\hline 17 & MYRIP & OA (up) & 4.77081124 \\
\hline 18 & SPARCL1 & OA (up) & 4.70336130 \\
\hline 19 & ABCA3 & OA (up) & 4.51342883 \\
\hline 20 & THBS1 & OA (down) & 4.29227205 \\
\hline
\end{tabular}

$\mathrm{FC}_{\mathrm{n}}, \mathrm{C}_{\mathrm{n}}$ and degree were transformed by $\mathrm{Z}$ core. Data of gene regulation was observed by using SAM4.0 (RA vs. OA). The threshold was set as log Ifold changel $\geq 2$. RA, rheumatoid arthritis, OA, osteoarthritis; INHBB; inhibin $\beta \mathrm{B}$; CPM, carboxypeptidase $\mathrm{M}$; ADH1C, alcohol dehydrogenase $1 \mathrm{C}$; ITGB2, integrin $\beta 2$; COL11A1, collagen, type XI, $\alpha$ 1; ADH1A, alcohol dehydrogenase 1A; C10orf116, chromosome 16 open reading frame 45 [Homo sapiens (human)]; SLC29A1, solute carrier family 29; PRG4, proteoglycan 4; CHL1, close homolog of L1; RARRES2, retinoic acid receptor responder protein 2; MS4A2, membrane-spanning 4-domains, subfamily A, member 2; CCR3, IFIT2, interferon-induced protein with tetratricopeptide repeats 2 ; RSAD2, radical S-adenosyl methionine domain containing 2; CLIC5, chloride intracellular channel 5; MYRIP, myosin VIIA and Rab interacting protein; SPARCL1, secreted protein, acidic and rich in cysteines-like 1; ABCA3, ATP-binding cassette, sub-family A, member 3; THBS1, thrombospondin 1 .

CCR3, radical S-adenosyl methionine domain containing 2 , thrombospondin 1 (THBS1) and membrane-spanning 4-domains, subfamily A, member 2, were intensively enriched in immune-associated biological process terms, including defense, inflammatory, immune responses, immune system process and response to wounding. In addition, ITGB2, THBS1, COL11A1, and close homolog of L1 were closely involved in cell adhesion and biological adhesion (Table III).

\section{Discussion}

Gene expression studies have been widely used to allow improved diagnosis and identify novel pathways implicated in the pathogenesis of autoimmune diseases. In the current study, DEGs in OA SFBs compared with RA SFBs were identified based on gene expression profiling, 1068 upregulated genes 
Table III. Top 20 disease candidate genes and related BP terms.

\begin{tabular}{llll}
\hline Rank & \multicolumn{1}{c}{ BP term } & $\mathrm{N}$ & \multicolumn{1}{c}{ Genes } \\
\hline 1 & Response to stimulus & 9 & $\begin{array}{l}\text { INHBB, PRG4, CCR3, RSAD2, MS4A2, } \\
\text { ITGB2, THBS1, COL11A1, ABCA3 } \\
\text { SLC29A1, MYRIP, CLIC5, MS4A2, ITGB2, } \\
2\end{array}$ \\
& Localization & 8 & THBS1, ABCA3, DNM1 \\
3 & Defense response & 6 & INHBB, CCR3, RSAD2, MS4A2, ITGB2, THBS1 \\
4 & Response to stress & 6 & INHBB, CCR3, RSAD2, MS4A2, ITGB2, THBS120 \\
5 & Response to external stimulus & 6 & INHBB, CCR3, MS4A2, ITGB2, THBS1, COL11A1 \\
6 & Immune system process & 5 & PRG4, RSAD2, MS4A2, ITGB2, THBS1 \\
7 & Cell adhesion & 5 & CCR3, ITGB2, THBS1, COL11A1, CHL1 \\
8 & Biological adhesion & 5 & CCR3, ITGB2, THBS1, COL11A1, CHL1 \\
9 & Immune response & 4 & PRG4, RSAD2, MS4A2, THBS1 \\
10 & Inflammatory response & 4 & CCR3, MS4A2, ITGB2, THBS1 \\
11 & Response to wounding & 4 & CCR3, MS4A2, ITGB2, THBS1 \\
12 & Inner ear morphogenesis & 2 & CLIC5, COL11A1 \\
\hline
\end{tabular}

Rank, the rank of BP term determined by the number of the 20 disease-related genes enriched in the BP term. N, number of the 20 disease-related genes involved in the BP term. BP, biological process; INHB2, inhibin $\beta$ B; PRG4, proteoglycan 4; RSAD2, radical S-adenosyl methionine domain containing 2; MS4A2, membrane-spanning 4-domains, subfamily A, member 2; ITGB2, integrin $\beta 2$; THBS1, thrombospondin 1; COL11A1, collagen, type XI, a 1; ABCA3, ATP-binding cassette sub-family A member 3; SLC29A1, solute carrier family 29; MYRIP, myosin-VIIa- and Rab-interacting protein; CLIC5, chloride intracellular channel 5; DNM1, dynamin 1; THBS120, thrombospondin 120.
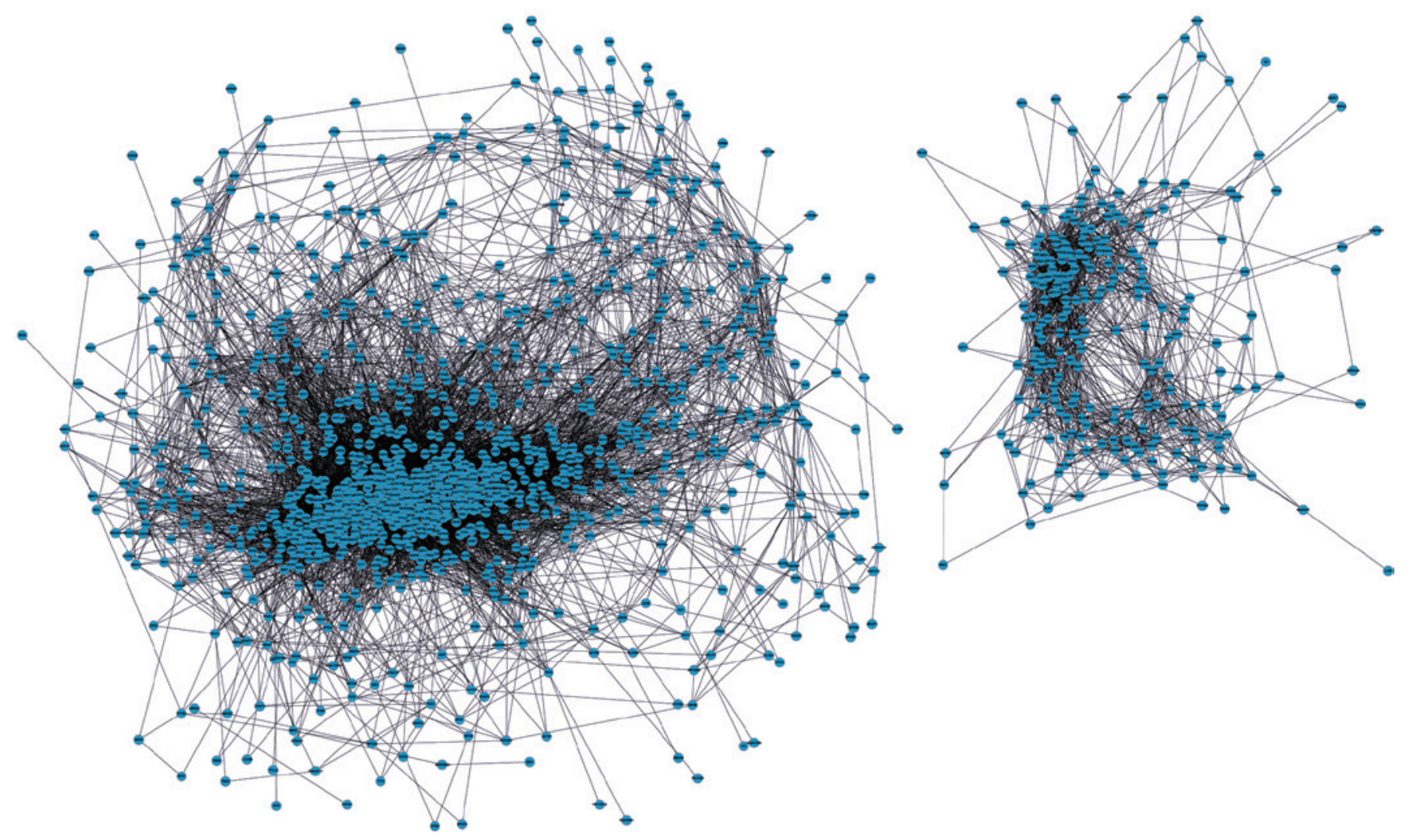

Figure 3. Differential gene co-expression network. A node represents one DEG (RA vs. OA) and is interconnected in the network. Co-expressed genes are linked by edges. DEGs, differentially expressed genes; RA, rheumatoid arthritis; OA, osteoarthritis.

and 245 downregulated genes were observed. Similarly, a previous study has reported different biological properties between RA and OA SFBs. Higher levels of specific cytokines were found in RA SFBs compared with OA SFBs, including epidermal growth factor, basic fibroblast growth factor, TGF- $\beta 1$, granulocyte-macrophage colony-stimulating factor, 
IL-1 $\beta$ and IL-6. In addition, by contrast with the OA SFBs, RA SFBs were observed to stimulate $\left[{ }^{3} \mathrm{H}\right]$ thymidine incorporation in the murine fibroblast cell line (25). Therefore, the current results support the hypothesis that RA and OA may result in the alterations of gene expression in the SFBs.

The development of an RA and OA gene co-expression network based on the topological analysis is critical, since it may provide visualized structural information regarding the connectivity of genes, compared with the traditional clustering analysis (26). Notably, the clustering coefficient and degree are two of the most important features of the network model. In the present study, the top 10 clustering coefficient $\mathrm{Cri}_{\mathrm{n}}$-associated genes were identified, which indicates a highly significant association between these genes and disease status. Notably, to the best of our knowledge the present study was the first to use the formula: $\mathrm{Cri}_{n}=\mathrm{FC}_{n}+$ degree $_{n}+\mathrm{C}_{n}$, which is comprised of fold change, average degree and clustering coefficient of co-expression gene network to calculate the top 20 disease-associated candidate genes.

For OA disease, Martin et al (27) reported that the single nucleotide polymorphism, rs2615977, located in intron 31 of COL11A1 (5th gene at ranking) is highly associated with OA. In addition, COL11A1 has been used as a significant target for musculoskeletal disease research (28). In the present study, COL11A1 was observed to be downregulated in OA samples compared with RA samples, which further supports the results of a previous study that suggested COL11A1 may have be associated with OA (29). In addition, the inhibition of cartilage hyperplasia appears to be another favorable approach to relieve symptoms of OA disease. Ruan et al (30) demonstrated that PRG4 (9th gene at ranking) inhibits cartilage metabolism and proliferation by upregulating hypoxia inducible factor-3 $\alpha$. In the current study, PRG4 was found to be upregulated in the OA group but downregulated in the RA group, which indicates that the PRG4 gene may have a potential association with the pathogenesis of RA and OA.

In RA, Rinaldi et al (31) suggested that $\beta 1$ integrins contribute to the tight binding of RA SFBs to the matrix and regulate extracellular matrix remodeling in the RA disease process in vivo. The function of $\beta 1$ integrins is similar to that of the ITGB2 identified in this study (4th gene at ranking). Notably, ITGB2 was identified to be upregulated in RA rather than OA, which further supports the hypothesis that ITGB2 may be critical in facilitating the RA disease process. INHBA and INHBB (1st gene at ranking) are two subunits of inhibin. El-Gendi et al (32) has demonstrated that the serum level of inhibin $\beta$ A was significantly higher in 60 patients with RA compared with 20 normal patients. In the present study, the expression level of INHBB was identified to be higher in RA compared with OA, indicating that INHBB may have a similar function to INHBA and may serve as a novel biological marker in the diagnosis of RA disease.

Furthermore, functional enrichment analysis of the top 20 disease-associated genes was performed to demonstrate the possible biological mechanisms underlying AR and OA, including response to stimulus, localization, response to stress, response to external stimulus and process involved in the development of the immune system. This finding is consistent with the results of previous studies, suggesting that the immune system is pivotal in auto-inflammatory and non-auto-inflammatory arthritis. The immune system regulates the alteration of cell osmosis and the inflammatory response caused by periostitis (33-36). The majority of the other top 20 disease-associated genes were enriched in terms of cell and biological adhesion. Notably, cell adhesion is also highly correlated with the pathogenesis of AR and OA. Karatay et al (37) stated that the decreased intercellular adhesion molecule-1 and vascular cell adhesion molecule-1 levels following intra-articular hyaluronic acid (HA) injection may aid in explaining the anti-inflammatory effects of HA therapy in OA of the knee.

In conclusion, the present study offers significant information that may aid in understanding the molecular mechanisms underlyng OA and RA. The top 20 disease-associated genes and associated BP terms were observed in this study, which may facilitate the design of targeted therapy for OA and RA in the near future.

\section{Acknowledgments}

This study was supported by the Shanghai Medical Key Subject Construction Project (ZK2012A28) and National Clinical Key Specialty Construction Project.

\section{References}

1. Maini R, Plater-Zyberk C and Andrew E: Autoimmunity in rheumatoid arthritis. An approach via a study of B lymphocytes. Rheum Dis Clin North Am 13: 319-338, 1987.

2. Buckwalter J and Mankin H: Articular cartilage: degeneration and osteoarthritis, repair, regeneration, and transplantation. Instr Course Lect 47: 487-504, 1997.

3. Brown RA and Weiss JB: Neovascularisation and its role in the osteoarthritic process. Ann Rheum Dis 47: 881-885, 1988.

4. Sattar A, Kumar P and Kumar S: Rheumatoid- and osteo-arthritis: quantitation of ultrastructural features of capillary endothelial cells. J Pathol 148: 45-53, 1986.

5. Keyszer G, Redlich A, Häupl T, et al: Differential expression of cathepsins B and L compared with matrix metalloproteinases and their respective inhibitors in rheumatoid arthritis and osteoarthritis: a parallel investigation by semiquantitative reverse transcriptase-polymerase chain reaction and immunohistochemistry. Arthritis Rheum 41: 1378-1387, 1998.

6. Ayral X, Pickering E, Woodworth T, Mackillop N and Dougados M: Synovitis: a potential predictive factor of structural progression of medial tibiofemoral knee osteoarthritis - results of a 1 year longitudinal arthroscopic study in 422 patients. Osteoarthritis Cartilage 13: 361-367, 2005.

7. Emery P, Breedveld FUC, Lemmel EM, et al: A comparison of the efficacy and safety of leflunomide and methotrexate for the treatment of rheumatoid arthritis. Rheumatology (Oxford) 39: 655-665, 2000.

8. Superio-Cabuslay E, Ward MM and Lorig KR: Patient education interventions in osteoarthritis and rheumatoid arthritis: a meta-analytic comparison with nonsteroidal antiinflammatory drug treatment. Arthritis Care Res 9: 292-301, 1996.

9. Ross C: A comparison of osteoarthritis and rheumatoid arthritis: diagnosis and treatment. Nurse Pract 22: 20-41, 1997.

10. Kofoed H and Sørensen TS: Ankle arthroplasty for rheumatoid arthritis and osteoarthritis: prospective long-term study of cemented replacements. J Bone Joint Surg Br 80: 328-332, 1998.

11. Wajon A, Carr E, Edmunds I and Ada L: Surgery for thumb (trapeziometacarpal joint) osteoarthritis. Cochrane Database Syst Rev: Oct 7, CD004631, 2009.

12. Silverstein FE, Faich G, Goldstein JL, et al: Gastrointestinal toxicity with celecoxib vs nonsteroidal anti-inflammatory drugs for osteoarthritis and rheumatoid arthritis: the CLASS study: a randomized controlled trial. Celecoxib Long-term Arthritis Safety Study. JAMA 284: 1247-1255, 2000.

13. Deeks JJ, Smith LA and Bradley MD: Efficacy, tolerability, and upper gastrointestinal safety of celecoxib for treatment of osteoarthritis and rheumatoid arthritis: systematic review of randomised controlled trials. BMJ 325: 619, 2002. 
14. Bramlage CP, Häupl T, Kaps C, et al: Decrease in expression of bone morphogenetic proteins 4 and 5 in synovial tissue of patients with osteoarthritis and rheumatoid arthritis. Arthritis Res Ther 8: R58, 2006.

15. Pohlers D, Beyer A, Koczan D, Wilhelm T, Thiesen HJ and Kinne RW: Constitutive upregulation of the transforming growth factor-beta pathway in rheumatoid arthritis synovial fibroblasts. Arthritis Res Ther 9: R59, 2007.

16. Bolstad BM, Irizarry RA, Astrand M and Speed TP: A comparison of normalization methods for high density oligonucleotide array data based on variance and bias. Bioinformatics 19: 185-193, 2003.

17. Tusher VG, Tibshirani R and Chu G: Significance analysis of microarrays applied to the ionizing radiation response. Proc Nat Acad Sci USA 98: 5116-5121, 2001.

18. Shannon P, Markiel A, Ozier O, et al: Cytoscape: a software environment for integrated models of biomolecular interaction networks. Genome Res 13: 2498-2504, 2003

19. Ruan J, Dean AK and Zhang W: A general co-expression network-based approach to gene expression analysis: comparison and applications. BMC Syst Biol 4: 8, 2010.

20. Tiret L, Rigat B, Visvikis S, et al: Evidence, from combined segregation and linkage analysis, that a variant of the angiotensin I-converting enzyme (ACE) gene controls plasma ACE levels. Am J Hum Genet 51: 197-205, 1992.

21. Schmitt WA Jr, Raab RM and Stephanopoulos G: Elucidation of gene interaction networks through time-lagged correlation analysis of transcriptional data. Genome Res 14: 1654-1663, 2004.

22. Welsh JB, Zarrinkar PP, Sapinoso LM, et al: Analysis of gene expression profiles in normal and neoplastic ovarian tissue samples identifies candidate molecular markers of epithelial ovarian cancer. Proc Natl Acad Sci USA 98: 1176-1181, 2001.

23. Huang da W, Sherman BT, Tan Q, et al: DAVID Bioinformatics Resources: expanded annotation database and novel algorithms to better extract biology from large gene lists. Nucleic Acids Res 35: W169-W175, 2007.

24. Aoki-Kinoshita KF and Kanehisa M: Gene annotation and pathway mapping in KEGG. Methods Mol Biol. 396: 71-91, 2007.

25. Bucala R, Ritchlin C, Winchester R and Cerami A: Constitutive production of inflammatory and mitogenic cytokines by rheumatoid synovial fibroblasts. J Exp Med 173: 569-574, 1991.
26. Schadt EE and Lum PY: Thematic review series: systems biology approaches to metabolic and cardiovascular disorders. Reverse engineering gene networks to identify key drivers of complex disease phenotypes. J Lipid Res 47: 2601-2613, 2006.

27. Martin S, Richards AJ, Yates J, Scott JD, Pope M and Snead MP. Stickler syndrome: further mutations in COL11A1 and evidence for additional locus heterogeneity. Eur J Hum Genet 7: 807-814, 1999.

28. Kahler RA, Yingst S, Hoeppner LH, et al: Collagen 11a1 is indirectly activated by lymphocyte enhancer-binding factor 1 (Lef1) and negatively regulates osteoblast maturation. Matrix Biol 27: 330-338, 2008.

29. Meulenbelt I: Osteoarthritis year 2011 in review: genetics. Osteoarthritis Cartilage 20: 218-222, 2012.

30. Ruan MZ, Erez A, Guse K, et al: Proteoglycan 4 expression protects against the development of osteoarthritis. Sci Transl Med 5: 176ra34, 2013.

31. Rinaldi N, Schwarz-Eywill M, Weis D, et al: Increased expression of integrins on fibroblast-like synoviocytes from rheumatoid arthritis in vitro correlates with enhanced binding to extracellular matrix proteins. Ann Rheum Dis 56: 45-51, 1997.

32. El-Gendi SS, Moniem AE, Tawfik NM, Ashmawy MM, Mohammed OA, Mostafa AK, Zakhari MM and Herdan OM: Value of serum and synovial fluid activin A and inhibin A in some rheumatic diseases. Int J Rheum Dis 13: 273-279, 2010.

33. Scanzello CR, Plaas A and Crow MK: Innate immune system activation in osteoarthritis: is osteoarthritis a chronic wound? Curr Opin Rheum 20: 565-572, 2008.

34. Sakkas LI and Platsoucas CD: The role of T cells in the pathogenesis of osteoarthritis. Arthritis Rheum 56: 409-424, 2007.

35. Feldmann M and Maini RN: Lasker Clinical Medical Research Award. TNF defined as a therapeutic target for rheumatoid arthritis and other autoimmune diseases. Nat Med 9: 1245-1250, 2003.

36. McInnes IB and Schett G: Cytokines in the pathogenesis of rheumatoid arthritis. Nat Rev Immunol 7: 429-442, 2007.

37. Karatay S, Kiziltunc A, Yildirim K, Karanfil RC and Senel K: Effects of different hyaluronic acid products on synovial fluid levels of intercellular adhesion molecule-1 and vascular cell adhesion molecule-1 in knee osteoarthritis. Ann Clin Lab Sci 34: $330-335,2004$ 